\title{
Boundary Condition for Dislocation Dynamic Simulation in BCC Crystal
}

\author{
Shuyang Dai ${ }^{1,2}$, Fengru Wang ${ }^{1}$, Yang Xiang $^{3}$, Jerry Zhijian Yang ${ }^{1,2, *}$ and \\ Cheng Yuan ${ }^{1}$ \\ ${ }^{1}$ School of Mathematics and Statistics, Wuhan University, Wuhan, 430072, China. \\ ${ }^{2}$ Hubei Key Laboratory of Computational Science, Wuhan University, Wuhan, 430072, \\ China. \\ 3 Department of Mathematics, Hong Kong University of Science and Technology, \\ Hong Kong, China.
}

Received 26 September 2020; Accepted 18 January 2021

\begin{abstract}
The movement of dislocations and the corresponding crystal plastic deformation are highly influenced by the interaction between dislocations and nearby free surfaces. The boundary condition for inclination angle $\theta_{\text {inc }}$ which indicates the relation between a dislocation line and the surface is one of the key ingredients in the dislocation dynamic simulations. In this paper, we first present a systematical study on $\theta_{\text {inc }}$ by molecular static simulations in BCC-irons samples. We also study the inclination angle by using molecular dynamic simulations. A continuum description of inclination angle in both static and dynamic cases is derived based on Onsager's variational principle. We show that the results obtained from continuum description are in good agreement with the molecular simulations. These results can serve as boundary conditions for dislocation dynamics simulations.
\end{abstract}

AMS subject classifications: 65G05, 70G75

Key words: Dislocation, dislocation dynamics, boundary conditions, analytical model, Onsager's variational principle.

\section{Introduction}

It is widely agreed that dislocations are the main carrier of plastic deformation in crystalline materials. The movement of dislocations is the key mechanism of strain energy relaxation which is highly influenced by the interaction with other defects, external loads and crystal structures [1-6]. Therefore it is important to note that the development of

*Corresponding author. Email addresses: shuyang_dai@whu.edu.cn (S. Dai), wangfr@whu.edu.cn (F. Wang), maxiang@ust.hk (Y. Xiang), zjyang.math@whu .edu.cn (J. Z. Yang), yuancheng@whu.edu.cn (C. Yuan) 
an accurate plasticity theory should based on the detailed dislocation mechanics that the movement of each dislocation could be traced, rather than on empirical assumptions $[1,7]$. However, as it is well-known that current models of dislocation need to consider phenomenon in multiple scales, i.e., the elastic interactions of dislocations are usually long range, but meanwhile, many other dislocation interactions such as self-force, annihilation, reaction, and multiplication are short range since these effects are highly depending on the local properties dislocation structure. Current simulation techniques which could track the movement of each dislocation in material are usually too complicated due to the multi-scale nature in the description of dislocations, therefore, more comprehensive models are needed to provide more accurate and efficient descriptions of the collective movement of dislocations.

During the last several decades, dislocation dynamics (DD) simulation, which can simulate the movements and interactions of large ensemble of dislocations by direct tracking the dynamics of individual discrete dislocation, were developed to deal with the complicated interactions as well as the pattern evolution of dislocations occurring in the plastic deformation [8-12]. DD simulation now is the most promising tool for the study of dislocations in crystalline materials in mesoscopic scale [13-41].

DD simulation have been successfully adopted to study various phenomenon in crystal plasticity at small scale, for instance, strain hardening effects in cubic or hexagonal crystals [22, 23, 30,38], microstructure in cyclic deformation [36], plasticity in polycrystalline materials [37]. However, in current conventional DD simulations, the periodic boundary condition is most useful boundary condition for simulation cell in order to calculate the stress due to dislocations based on stress expression in infinite medium [15], it is still a non-trivial task for DD simulations to account the effects of various boundary conditions [42]. How to account the effects induced by various boundaries such as free surfaces in simulations accurately and efficiently is still a crucial problem in the developing of sophisticated DD simulations.

The effect of boundaries such as free surfaces is introduced by the image stress. The image stress is an additional stress in order to guarantee the traction-free boundary condition, which can be calculated based on instantaneous dislocation configuration [1]. In DD simulations, the movements of dislocation segments are determined by the total stress including the image stress, the self stress, the stress due to dislocations and other defects, and the external applied stress. One general approach [13] adopted in calculating the total stress is that the stress is decomposed into two parts, one is the stress due to dislocation ensemble in infinite medium which could be obtained based on classical dislocation theory, and the other one comes from the effects of boundary conditions, i.e., the image stress, which could be solved by using some continuum theories such as finite element method (FEM) or boundary element method (BEM) [13, 17-19, 25, 29,34]. Many models derived from this approach have been widely used in the study of crystal plasticity [31,39-41]. These models work well when dislocation segments are relatively away from the free surfaces. However, the image stress due to the boundary are singular when these dislocations approach to and/or intersect with the free surfaces [14,21,25,31,40,42], 
which leads to the failure of continuum assumption in these theories. Therefore the resultant behavior of dislocations based on these theories are questionable in these cases.

Several attempts have been made to overcome this problem by eliminating the effects of singularity in calculation of image stress near the surface. For instance, a numerical method based on adaptive mesh with multiple resolutions was used to approximate the divergent behavior of stress field near the intersection point [21], and the authors also suggested an approximation method to calculate the image stress at the intersection point by replacing the actual singular stress with an average value on the numerical grids in a vicinity region of boundary. In [25], the singularity was removed by a decomposition and superposition procedure, in which the actual intersecting dislocation is considered as a superposition of two dislocations: one is a straight dislocation in the elastic half-space, this straight dislocation intersects with the surface in an angle equivalent the intersection angle of actual dislocation, and the image stress due to these straight dislocation could been obtained by Yoffe [43] analytically; another dislocation can be viewed as the residual of actual dislocation minus the previous straight dislocation in elastic half-space, and there are no singularity in the calculation of the stress due to this residual dislocation. Another work done in [31] adopted a similar decomposition approach to eliminate the singularity, in their method, image force was calculated in Fourier space with the help of virtual dislocation segments. Once the image stress was obtained, the relation between dislocation and surface, i.e., the inclination angle could be determined by the force balance at the intersection node.

A more simple and direct approach trying to overcome the problem due to singularity is that the inclination angle between dislocation and free surface is specifying explicitly as a boundary condition and this boundary condition could be directly used in DD simulations. An explicit analytical expression of the boundary condition for straight dislocation in isotropic medium was firstly given by Lothe [42], in which the straight dislocation intersects with the surface at a certain inclination angle. Schwarz [14] proposed a simplified boundary condition for symmetric case that dislocation normally enters the surface. This normal assumption is not a correct one in general cases since the inclination angle between dislocation and boundary is barely equal to $90^{\circ}$, however it was also widely adopted in the simulations of dislocation dynamics due to the fact that this boundary condition can still provide some correct insights in some DD simulations as shown in Refs. [18, 27, 28]. In Ref. [14], Schwarz also suggested that the boundary condition proposed by Lothe should be used in asymmetric cases. The same boundary condition is rediscovered in [31] based upon line tension model in thin film with the assumption of straight line approximation. The authors verified this analytical boundary condition by comparing the results with those obtained in DD simulations and molecular static simulations in thin film (about $10 \sim 20 \mathrm{~nm}$ ). It can be found that this boundary condition can be used in static simulations within isotropic medium and the slip plane of dislocation is normal to the free surface. However, since the detailed interactions between the dislocation core and the surface can only be fully considered on atomic level [42], it is very important to note that the validity of those derived analytical relations should be verified 
systematically by both molecular static and dynamic simulations considering the effects of various surface normal, temperature and dislocation configurations.

In the present work, we first study the inclination angle $\theta_{\text {inc }}$ between dislocation and surface by using both static and dynamic molecular simulations systematically. A general expression for $\theta_{\text {inc }}$ is derived based upon Onsager's variational principle. The results obtained based on this generalized boundary condition agree well with the results obtained in both static and dynamic simulations for various atomistic simulation samples. From the results of atomistic simulations, a simplified evolution model is further derived based upon straight line approximation.

\section{Molecular simulations on the dislocation boundary condition}

In present work, we first perform molecular simulations on body-centered crystal $\alpha$-iron to study the inclination angle $\theta_{\text {inc }}$ systematically.

The molecular simulations in this work are performed by using EAM-type potential for $\alpha$-iron, which is developed by Mendelev and co-workers [44]. This potential was constructed by fitting a proposed functional form to perfect crystal and crystal defects data as well as experimental liquid structure factor data. The EAM-type potential had been widely used to study various properties in $\alpha$-iron such as the glide of screw dislocation motion [45] and the interactions of carbon atoms with dislocation [46]. The molecular simulations in our work are performed in NVT ensemble.

The notations used in this work are shown in Fig. 1 . Here $\vec{n}$ is the normal of the free surface, $\vec{n}_{1}$ is the unit projection vector of surface normal $\vec{n}$ onto the slip plane, $\vec{n}_{1}=\vec{n}$ when the slip plane is perpendicular to the free surface. $\theta_{0}$ and $\theta_{b}$ are the angles between surface normal $\vec{n}$ and Burgers vector $\vec{b}$ with respect to the projected direction of surface normal $\vec{n}_{1}$, respectively. $\alpha$ and $\beta$ are the angles between $\vec{n}_{1}$ and $\vec{b}$ with respect to the dislocation line direction $\vec{\xi}$, i.e., $\cos \alpha=\left|\overrightarrow{\boldsymbol{n}}_{1} \cdot \overrightarrow{\boldsymbol{\xi}}\right| /\left(\left|\overrightarrow{\boldsymbol{n}}_{1}\right| \cdot|\overrightarrow{\boldsymbol{\xi}}|\right)$ and $\cos \beta=|\overrightarrow{\boldsymbol{b}} \cdot \vec{\xi}| /(|\overrightarrow{\boldsymbol{b}}| \cdot|\overrightarrow{\boldsymbol{\xi}}|)$, respectively. The value of $\alpha$ and $\beta$ varies during the glide of the dislocation, but their summation should be $\theta_{b}$.

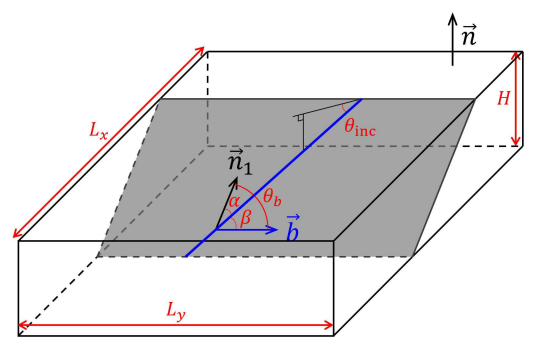

Figure 1: The initial cell configuration. $\vec{n}$ is the surface normal of the thin film, $\vec{n}_{1}$ is the projection of the surface normal onto the slip plane, $\vec{b}$ is the Burgers vector. The blue line is the dislocation, and the shaded region represents the slip plane. 


\subsection{Simulation cell setup in molecular simulations}

In order to study the inclination angle between the dislocation and the free surface systematically, a series of large initial cubic boxes are generated with different types of dislocations, including edge, screw and mixed dislocations. Each large initial cubic box contains a dislocation dipole with opposite Burgers vectors in order to satisfy periodic boundary condition of this cubic box. The Burgers vectors of these two dislocations are $\vec{b}=1 / 2[111]$ and $\vec{b}=-1 / 2[111]$, respectively. Since the edge and the mixed dislocations in body-centered crystals such as $\alpha$-iron are either in $\{110\}$ slip system or in $\{112\}$ slip system when the temperature is not very high, the slip plane of the generated initial dislocation could be either (11̄0) plane or $(11 \overline{2})$ plane. Therefore five cubic boxes are constructed and the types of dislocation configurations contained in these cubic boxes are summarized in Table 1. These cubic boxes can be categorized into two cases based on types of the initial dislocations: 1. Initial edge or mixed dislocation on particular slip plane $((1 \overline{1} 0)$ or $(11 \overline{2})) ; 2$. Initial screw dislocation. It is worthy to note that the number of atoms containing in these large initial cubic boxes are about several tens millions, it is large enough in our work to study the boundary condition of dislocations.

All initial cubic boxes are pre-relaxed in MD simulations at temperature $T=100 \mathrm{~K}$ for $10 \mathrm{~ns}$ and then quenched to $T=0 \mathrm{~K}$ in order to find the equilibrium atomic structure of the dislocation dipole in all five cubic boxes. Following these steps, the metastable states are less likely to be trapped in MD simulations.

After the equilibrium dislocation structures are obtained, the initial simulation cells are generated by cutting these cubic boxes. The resultant cell contains only one threading dislocation intersecting with two parallel free surfaces. Several initial simulation cells are cut from one cubic box with different surface normal. The free boundary condition is used in our molecular simulations for all six surfaces of the initial simulation cell.

In molecular static simulations, all simulation cells are relaxed at constant temperature $300 \mathrm{~K}$ for $52 \mathrm{~ns}$ simulations using a time step of $0.0013 \mathrm{ps}$. The temperature are enforced in static simulations to guarantee that dislocations are not trapped in some metastable states. After discarding the first 40ns to allow for the dislocation reaching the equilibrium position, the inclination angle for each simulation cell is calculated based upon the quenched atomic structure at the simulation time 40ns, 46ns and 52ns, respectively. It is worthy to note that in the determination of inclination angles, we first get the core atoms of dislocation based on the rescaled centrosymmetry parameter between 0.05 and 0.4 , then the continuum dislocation line can be obtained as the central line of all these core atoms. The inclination angle can be calculated as the intersection angle between continuum dislocation line and surface. The final resultant inclination angle is the average value of the inclination angle at those three times.

In molecular dynamics simulations, all initial simulation cells are deformed by external constant strain $\varepsilon_{0}=0.25 \%$ and $\varepsilon_{0}=0.5 \%$ applied on the upper and lower free surfaces. The time step of MD simulations is $0.0013 \mathrm{ps}$. The atomic configurations are relaxed at different temperatures $T_{1}=300 \mathrm{~K}$ and $T_{2}=500 \mathrm{~K}$ to investigate the temperature effect. 


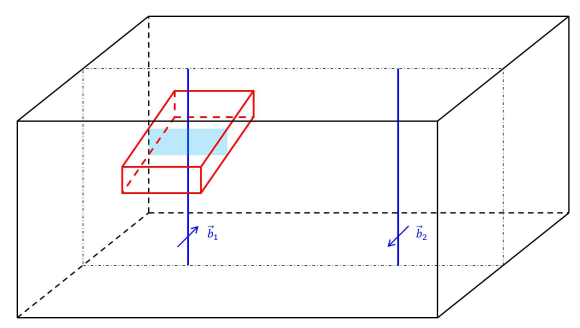

Figure 2: Cutting the initial simulation cell from the pre-relaxed large enough cubic box. The blue lines represent the dislocations and the red box represent the generated initial simulation cell. The shaded area is the slip plane.

Table 1: Information of five initial dislocation configurations. The Burgers vector of all dislocations is $\vec{b}=\frac{1}{2}[111]$. For a screw dislocation, the slip plane cannot be specified.

\begin{tabular}{||ccccc||}
\hline Case & Type & $\vec{\xi}_{\text {init }}$ & Slip plane & $\theta_{b}$ \\
\hline 1 & Edge & {$[11 \overline{2}]$} & $(1 \overline{1} 0)$ & $90^{\circ}$ \\
2 & Mixed & {$[110]$} & $(1 \overline{1} 0)$ & $35.3^{\circ}$ \\
3 & Edge & {$[1 \overline{1} 0]$} & $(11 \overline{2})$ & $90^{\circ}$ \\
4 & Mixed & {$[201]$} & $(11 \overline{2})$ & $39.2^{\circ}$ \\
5 & Screw & {$[111]$} & & $0^{\circ}$ \\
\hline
\end{tabular}

\subsection{Static simulations for initial edge/mixed dislocations}

We first study the static atomistic structure and calculate the projected inclination angle $\alpha$ of equilibrium dislocations for initial simulation cells containing an edge/mixed dislocation on slip plane (1110). The surface normal of the simulation cells are [111], [221], [110], $[22 \overline{1}],[11 \overline{1}],[22 \overline{3}]$ and $[11 \overline{2}]$, respectively. In order to eliminate the size effects due to the finite width and surface area of the simulation cell, it is important to generate multiple simulation cells with the same surface normal but in different widths and surface areas. In this work, four different initial simulation cells are generated, and the dimensions of these four cells for each surface normal are listed in Table 2. After verifying the convergence of the projected inclination angle in these four cells, the resultant value could be obtained by calculating the average of the angles measured in these four samples.

Fig. 3(a1)-(a7) show the equilibrium atomic configurations for the initial edge/mixed dislocation within slip plane $(1 \overline{1} 0)$ in the cases that the slip plane is perpendicular to the surface. The atomistic results show that the dislocation lines are almost perfectly straight in all simulations and the equilibrium orientations are independent of the initial dislocation type. The detailed values of the equilibrium projected inclination angle $\alpha$ are shown in Table 3 and also shown in Fig. 4 by the blue dots.

We also performed static simulations for initial edge/mixed dislocation within slip

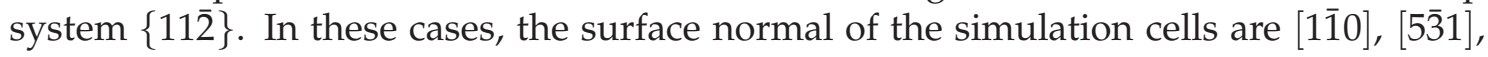
[201], [312] and [423], respectively. The conclusions are the same as previous simulations 
Table 2: The dimensions of initial simulation cell. $H$ is the width of the simulation cell, and $L_{\min }$ is the smaller value in $L_{x}$ and $L_{y}$. In our simulations, there are only small different in $L_{x}$ and $L_{y}$ due to the cutting procedure. $H, L_{x}$ and $L_{y}$ can be found in Fig. 1 in the main text. (Unit: nm)

\begin{tabular}{||cccccc||}
\hline$\vec{n}$ & & Sample 1 & Sample 2 & Sample 3 & Sample 4 \\
\hline \multirow{2}{*}[111]{} & $H$ & 11 & 11 & 21 & 21 \\
& $L_{\min }$ & 16 & 28 & 16 & 28 \\
\hline \multirow{2}{*}[221]{} & $H$ & 11 & 11 & 21 & 21 \\
& $L_{\min }$ & 17 & 30 & 17 & 30 \\
\hline \multirow{2}{*}[110]{} & $H$ & 11 & 11 & 21 & 21 \\
& $L_{\min }$ & 12 & 24 & 12 & 24 \\
\hline \multirow{2}{*}[22\overline{1}]{} & $H$ & 11 & 11 & 21 & 21 \\
& $L_{\min }$ & 13 & 27 & 13 & 27 \\
\hline \multirow{2}{*}[11\overline{1}]{} & $H$ & 11 & 11 & 21 & 21 \\
& $L_{\min }$ & 16 & 28 & 16 & 28 \\
\hline \multirow{2}{*}[22\overline{3}]{} & $H$ & 11 & 11 & 21 & 21 \\
& $L_{\min }$ & 16 & 28 & 16 & 28 \\
\hline \multirow{2}{*}[11\overline{2}]{} & $H$ & 11 & 11 & 21 & 21 \\
& $L_{\min }$ & 16 & 28 & 16 & 28 \\
\hline
\end{tabular}

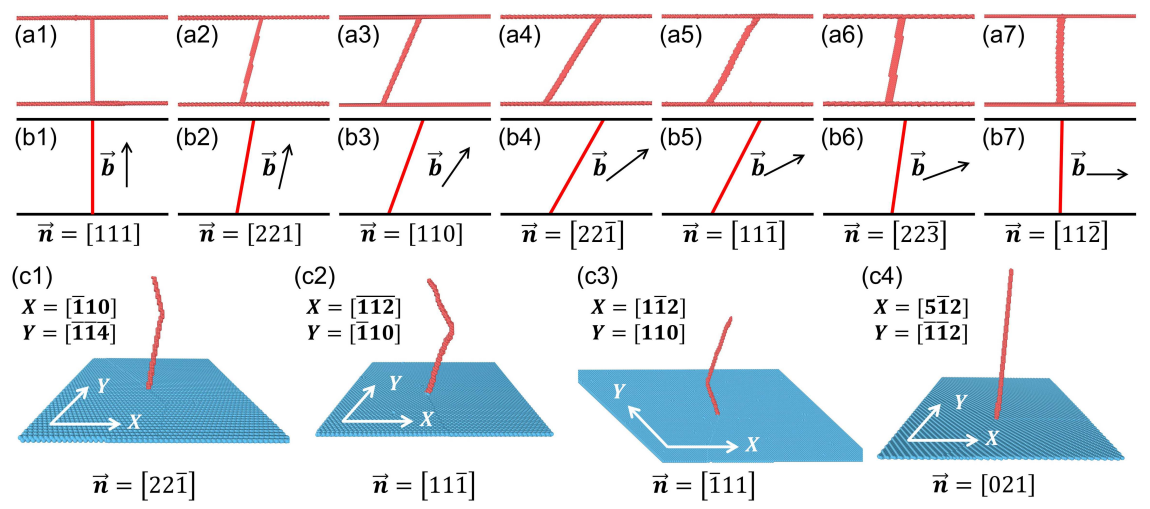

Figure 3: Comparisons of the results of molecular simulations and the analytical model. (a1)-(a7) are the equilibrium states obtained by molecular simulations. Only surface atoms and the atoms in dislocation core are shown. (b1)-(b7) are the results obtained from our analytical expression. (c1)-(c4) are the results for initial screw dislocation. Only lower surface atoms and the dislocation core atoms are shown.

that equilibrium dislocations are almost straight and their directions are independent on initial dislocation types. The detailed values of the equilibrium projected inclination angle $\alpha$ are shown in Table 4 and also shown by the red dots in Fig. 4 . 

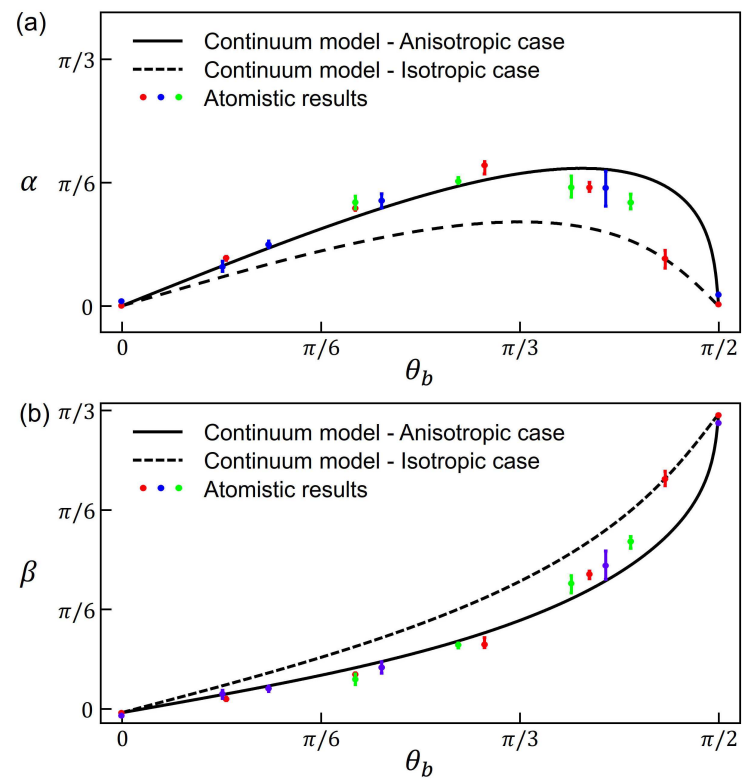

Figure 4: The results of (a) the projected inclination angle $\alpha$ and (b) the angle between dislocation and its Burgers vector $\beta$. The solid lines are the results from anisotropic elasticity and dashed lines are results from isotropic elasticity. The dots are the results from atomistic calculations.

Table 3: The projected inclination angle $\alpha$ for initial edge and mixed dislocation on slip plane (11̄0). (Unit: $\mathrm{rad})$

\begin{tabular}{||cccccc||}
\hline \multirow{2}{*}{$\vec{n}$} & $\overrightarrow{\boldsymbol{\xi}}_{\text {init }}$ & Sample 1 & Sample 2 & Sample 3 & Sample 4 \\
\hline \multirow{2}{*}[111]{} & edge & 0.00 & 0.00 & 0.00 & 0.00 \\
& mix & 0.00 & 0.00 & 0.00 & 0.00 \\
\hline \multirow{2}{*}[221]{} & edge & 0.21 & 0.19 & 0.20 & 0.20 \\
& mix & 0.21 & 0.20 & 0.20 & 0.20 \\
\hline \multirow{2}{*}[110]{} & edge & 0.42 & 0.43 & 0.42 & 0.41 \\
& mix: & 0.43 & 0.42 & 0.43 & 0.42 \\
\hline \multirow{2}{*}[22\overline{1}]{} & edge & 0.63 & 0.60 & 0.57 & 0.57 \\
& mix & 0.58 & 0.60 & 0.56 & 0.58 \\
\hline \multirow{2}{*}[11\overline{1}]{} & edge & 0.54 & 0.50 & 0.53 & 0.50 \\
& mix & 0.53 & 0.51 & 0.52 & 0.51 \\
\hline \multirow{2}{*}[22\overline{3}]{} & edge & 0.17 & 0.18 & 0.17 & 0.16 \\
& mix & 0.18 & 0.19 & 0.19 & 0.22 \\
\hline \multirow{2}{*}[11\overline{2}]{} & edge & 0.06 & 0.05 & 0.04 & 0.02 \\
& mix & 0.04 & 0.05 & 0.06 & 0.02 \\
\hline
\end{tabular}


Table 4: The projected inclination angle $\alpha$ for initial edge and mixed dislocation on slip plane (112̄). (Unit: rad)

\begin{tabular}{||cccccc||}
\hline \multirow{2}{*}{$\vec{n}$} & \multirow{2}{*}{$\vec{\xi}_{\text {init }}$} & Sample 1 & Sample 2 & Sample 3 & Sample 4 \\
\hline \multirow{2}{*}[1\overline{1}0]{} & edge & 0.00 & 0.00 & 0.00 & 0.01 \\
& mix & 0.02 & 0.02 & 0.03 & 0.01 \\
\hline \multirow{2}{*}[5\overline{3}1]{} & edge & 0.21 & 0.19 & 0.20 & 0.19 \\
& mix & 0.21 & 0.20 & 0.20 & 0.19 \\
\hline \multirow{2}{*}[201]{} & edge & 0.46 & 0.44 & 0.42 & 0.43 \\
& mix & 0.57 & 0.56 & 0.54 & 0.56 \\
\hline \multirow{2}{*}[312]{} & edge & 0.27 & 0.26 & 0.26 & 0.27 \\
& mix & 0.25 & 0.26 & 0.24 & 0.24 \\
\hline \multirow{2}{*}[423]{} & edge & 0.19 & 0.19 & 0.19 & 0.20 \\
& mix & 0.17 & 0.18 & 0.19 & 0.18 \\
\hline
\end{tabular}

\subsection{Static simulations for initial screw dislocations}

For the simulation cells containing an initial screw dislocation, since we have found that the finite size effects can be eliminated in the largest sample in previous simulations, the simulations in this subsection will be performed in the largest sample. In this work, we generated various initial simulation cells with different surface normals including $[7 \overline{5} 1],[5 \overline{3} 1],[201],[021],[11 \overline{1}],[\overline{1} 11],[1 \overline{1} 1]$ and $[22 \overline{1}]$, respectively. The relaxation results show that in some cells, the final equilibrium dislocations are still one single straight lines (as shown in Fig. 3(c4)), but in other simulation cells, the equilibrium dislocations are no longer single straight lines but will break into two straight segments (as shown in Fig. 3(c1)-(c3)). Based upon these observations, we categorize the simulation cells into two groups base on their surface normals as follows.

The first group includes simulation cells with the former four surface normals (i.e., [751] , [53̄1], [201] and [021]). The planes formed by these surface normals and the Burgers vectors belong to (110), and their equilibrium dislocations are single lines. The second

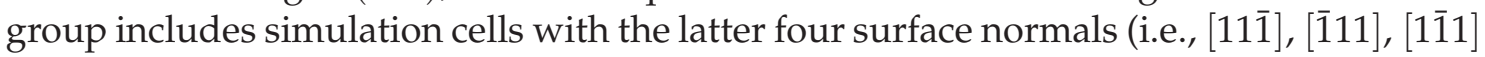
and $[22 \overline{1}])$. The planes formed by these surface normal and the Burgers vectors belong to (112), and the dislocations in these simulation cells will break into two segments. These two dislocation segments stay in different slip planes but the slip plane of both dislocations belongs to $\{112\}$ slip system. We can also find that the inclination angles for these two segments are the same. The results of the equilibrium $\alpha$ for each segment in all simulations are shown in Fig. 4 by green dots. The detailed value of the measured projected inclination angle $\alpha$ are shown in Table 5 and Table 6 , respectively.

Since the perfect screw dislocation has an infinite number of possible slip planes and the preferred slip planes for an equilibrium dislocation with edge component are either 
Table 5: The projected inclination angle $\alpha$ for initial screw dislocation with surface normal slip [75̄1], [53̄1], [201] and [021]. (Unit: rad)

\begin{tabular}{||lllll||}
\hline$\vec{n}$ & {$[7 \overline{5} 1]$} & {$[5 \overline{3} 1]$} & {$[201]$} & {$[021]$} \\
\hline $\bar{\alpha}$ & 0.439 & 0.479 & 0.460 & 0.440 \\
\hline
\end{tabular}

Table 6: The projected inclination angle $\alpha$ for initial screw dislocation with surface normal slip [111]], [1]11], $[1 \overline{1} 1]$ and $[22 \overline{1}]$. (Unit: rad)

\begin{tabular}{||cccccc||}
\hline$\vec{n}$ & Segment No. & {$[11 \overline{1}]$} & {$[\overline{1} 11]$} & {$[1 \overline{1} 1]$} & {$[22 \overline{1}]$} \\
\hline $\bar{\alpha}$ & 1 & 0.520 & 0.493 & 0.473 & 0.535 \\
& 2 & 0.537 & 0.470 & 0.547 & 0.525 \\
\hline
\end{tabular}

$\{110\}$ or $\{112\}$, the resultant slip plane depends on the local stress generated by initial configurations and applied to the initial perfect screw dislocation. We find that the resultant slip planes of the equilibrium dislocation in all simulations performed in this subsection are not perpendicular to the surface, therefore the results here can also be used to show the relationship of the projected inclination angle when the slip plane is not perpendicular to the free surface.

\subsection{Dynamics simulations in deformed cells}

In dynamic simulations, a strain $\varepsilon_{0}$ is applied to simulation samples in order to initiate the glide motion of dislocations. The effects of different surface normal and applied strains are studied and the results are shown in Fig. 5(a)-(b) (for different surface normal) and Fig. 6(a)-(b) (for different applied strains). In Fig. 5(a)-(b), the dynamic simulations are performed under temperature $T=300 \mathrm{~K}$. It can be found that dislocations in different samples with various directions of surface normal and applied strains have almost the same behavior: The inclination angle in dynamics will approach to the static equilibrium orientation obtained in previous static simulations in the beginning, and then the entire dislocation will glide as a straight line under the external shear strain. The values of the inclination angle $\theta_{\text {inc }}$ during dynamics are almost equal to the corresponding static inclination angles. The time of the initial rotation stage is short. $(\approx 10 \mathrm{ps}$ as shown in Fig. 5(a2)-(a4), (b2)-(b4) and also Fig. 6(a2)-(a5), (b2)-(b5)). Since the velocities of dislocations in metals are in the range from several tens to thousands meters per second $[47,48]$, the displacement of the dislocation during this short time is small (usually less than several nano-meters). After this initial short rotation stage, the value of $\theta_{\text {inc }}$ in the dynamic process is almost the same as the corresponding value in the static case. This can be clearly seen from Fig. 5(c), in which the instantaneous inclination angles measured during dynamic processes for dislocations in different slip planes (i.e., [110] or [112]) in a relatively long time only have slight deviations from a constant value, which is the value of inclination angle $\theta_{\text {inc }}$ obtained in static simulations. 

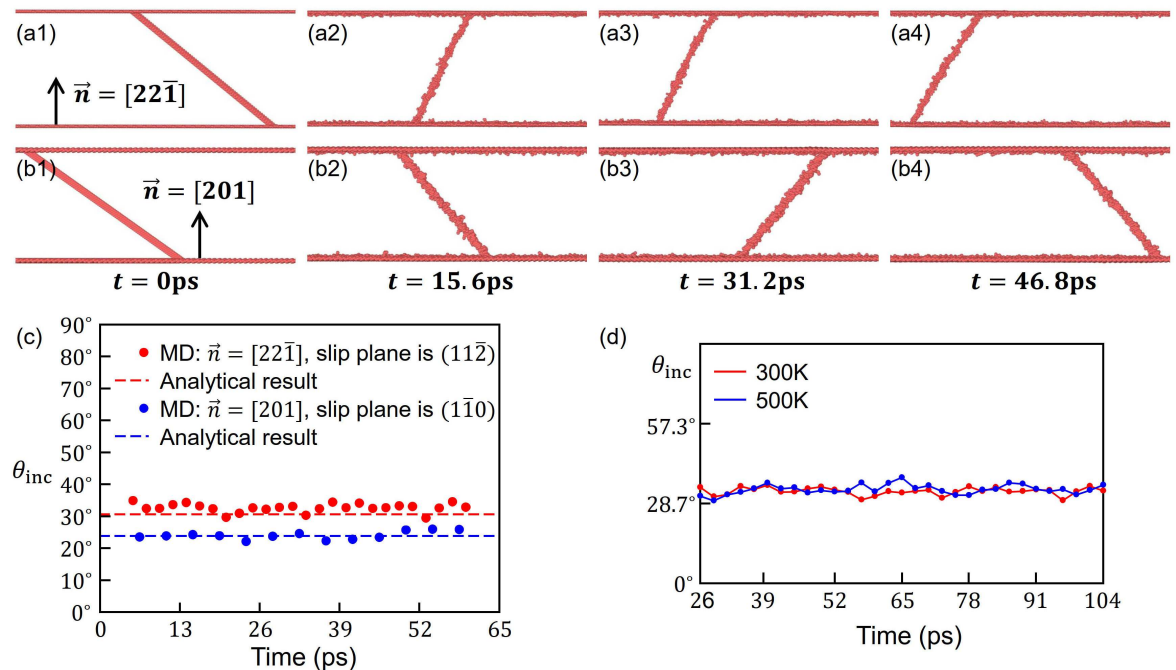

(d)

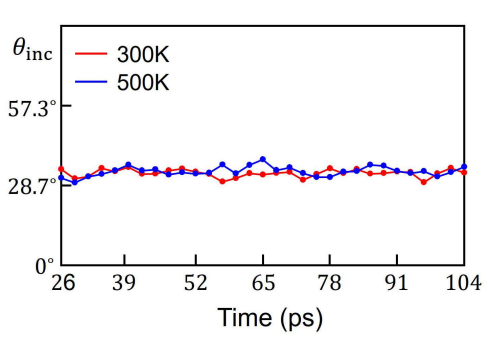

Figure 5: Results of dynamic simulations. (a) and (b) show the snapshots in the dynamic simulation of an initial edge dislocation at different simulation time with various surface normal of samples. (a) $\vec{n}=[22 \overline{1}]$ and (b) $\vec{n}=[201]$. The four figures from (a1) to (a4) (and also for (b1) to (b4)) are the snapshots at time $t=0 \mathrm{ps,}$ $t=15.6 \mathrm{ps}, t=31.2 \mathrm{ps}$ and $t=46.8 \mathrm{ps}$, respectively. The red dots represent the atoms in dislocation core and the free surfaces. (c) Results of the dynamic inclination angle measured in samples with different surface normals $[201]$ and $[22 \overline{1}]$. The dashed lines represent the values obtained by analytical boundary condition. (d) The effect of temperature on the projected inclination angle.

We also examine the temperature effect on the dynamic inclination angle by varying $T$ from $300 \mathrm{~K}$ to $500 \mathrm{~K}$. The comparisons of the measured inclination angle are shown in Fig. 5(d), which imply that the temperature has little effect on the averaged value of the inclination angle $\theta_{\text {inc }}$. These observations show that the static inclination angle can be directly used in dynamic process as a boundary condition in different temperatures.

\section{Analytical boundary condition for $\theta_{\text {inc }}$}

In this section, an analytical model for both the static and dynamic inclination angle will be derived and compared with previous molecular simulations. We begin the derivation by reviewing the dislocation velocity that depends on the Peach-Koehler force $\vec{f}_{\text {Peach-Koehler }}$ acting on the dislocation [1,2]:

$$
\overrightarrow{\boldsymbol{v}}=M_{d} \vec{f}_{\text {Peach-Koehler }}=M_{d}\left(\left(\sigma^{\mathrm{d}}+\sigma^{\mathrm{i}}+\sigma^{\mathrm{ext}}\right) \cdot \overrightarrow{\boldsymbol{b}}\right) \times \overrightarrow{\boldsymbol{\xi}},
$$

where $\sigma^{\mathrm{d}}, \sigma^{\mathrm{i}}$ and $\sigma^{\text {ext }}$ are the dislocation self stress, the image stress and the external applied stress on dislocation, respectively.

The force on the dislocation line at a point very close to the intersection point on the free surface was derived analytically in Ref. [42] as $d F=\frac{1}{\lambda}\left(-\frac{E_{0}(\beta)}{\tan \theta_{\text {inc }}}+\frac{d E_{0}(\beta)}{d \theta_{\text {inc }}}\right)$, where $\lambda$ is the distance from the surface intersection point, $E_{0}(\beta)$ is the prelogarithmic energy factor of 

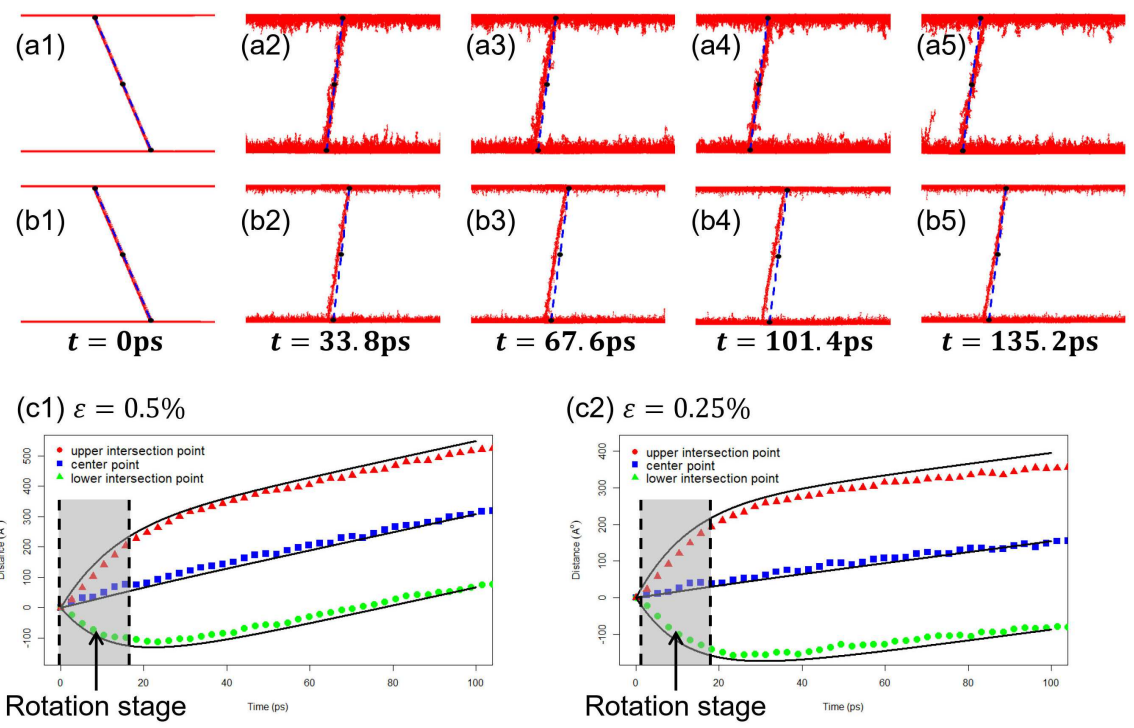

Figure 6: (a) and (b) show the snapshots of the dynamics of an initial edge dislocation gliding in the slip plane $(11 \overline{2})$ under different values of the applied strain: (a) $\varepsilon_{0}=0.5 \%$ and (b) $\varepsilon_{0}=0.25 \%$, respectively. The five figures from (a1) to (a5) (and also for (b1) to (b5)) are the snapshots at time $t=0 \mathrm{ps}, t=33.8 \mathrm{ps}, t=67.6 \mathrm{ps}, t=101.4 \mathrm{ps}$ and $t=135.2 \mathrm{ps}$, respectively. The red dots represent the atoms in the dislocation core and the free surfaces, and the dashed blue lines are the analytical results of the simplified model based upon straight line approximation. (c) The displacements of the upper intersection point, the center point and the lower intersection point of the dislocation during the dynamic simulations. The positions of these points are label by black dots in (a) and (b).

the dislocation which depends on the angle between the dislocation line and its Burgers vector, i.e., $\beta$ as shown in Fig. 1. If the term $\left(-\frac{E_{0}(\beta)}{\tan \theta_{\text {inc }}}+\frac{d E_{0}(\beta)}{d \theta_{\text {inc }}}\right) \neq 0$, the image stress will diverge as $\lambda$ approaches to zero, i.e., gets closer to the intersection point. This implies that [42]:

$$
-\frac{E_{0}(\beta)}{\tan \theta_{\text {inc }}}+\frac{d E_{0}(\beta)}{d \theta_{\text {inc }}}=0
$$

This equation specifies the boundary condition for a dislocation when it intersects with the free surface. However, it was derived when the dislocation slip plane is normal to the surface and only applies to this special case.

In a general case, the slip plane of the dislocation may not necessarily be perpendicular to the free surface. We modify Eq. (3.2) by using the relation $\sin \theta_{\text {inc }}=\cos \alpha \cdot \cos \theta_{0}$ that

$$
\frac{-\cos \theta_{\text {inc }}}{\cos \theta_{0} \sin \alpha}\left(E_{0}(\beta) \tan \alpha+\frac{d E_{0}(\beta)}{d \alpha}\right)=0
$$

That is, this condition is derived in terms of the projected inclination angle $\alpha$ instead of $\theta_{\text {inc. }}$ 
The explicit form of $\alpha$ depends on the expression of prelogarithmic energy factor $E_{0}(\beta)$. In both isotropic and anisotropic media, the perlogarithmic energy factor is $E_{0}(\beta)=$ $\frac{K_{e}}{4 \pi}\left(b_{\mathrm{e}}^{2}+k b_{\mathrm{s}}^{2}\right)$, where $b_{\mathrm{e}}=|\overrightarrow{\boldsymbol{b}}| \sin \beta$ and $b_{\mathrm{s}}=|\overrightarrow{\boldsymbol{b}}| \cos \beta$ are the edge and screw components of the Burgers vector of the dislocation, $K_{e}$ is the energy factor for pure edge dislocation and $k$ is the ratio of the energy factor between a pure screw dislocation and a pure edge dislocation. In an isotropic medium, $K_{e}=\frac{\mu}{1-v}$ and $k=1-v$, where $\mu$ and $v$ are the shear modulus and Poisson's ratio. In an anisotropic medium, the expressions of $K_{e}$ and $k$ in a BCC crystal can be derived by using the method of Eshelby [49], which gives that

$$
\begin{aligned}
& k=\frac{2\left(c_{11} c_{44}-c_{15}^{2}\right)}{c_{11}^{2}-c_{12}^{2}}, \\
& K_{e}=\frac{\left(c_{11}+c_{12}\right)\left(c_{66} c_{44}-c_{15}^{2}\right) \sqrt{\left(c_{11} c_{44}-c_{15}^{2}\right)\left(c_{11}-c_{12}\right)}}{\left(c_{11} c_{44}-c_{15}^{2}\right) \sqrt{\left(c_{66} c_{44}-c_{15}^{2}\right)\left(c_{11}-c_{12}\right)+2\left(c_{11}+c_{12}\right)\left(c_{66} c_{44}-c_{15}^{2}\right)}},
\end{aligned}
$$

where $c_{i j}$ are the elastic constants of BCC crystal in the coordinate system that the direction of $x-, y$ - and $z$-axis are $[\overline{1} 2 \overline{1}],[\overline{1} 01]$ and [111], respectively.

It can be seen that the energy factor of screw dislocation is smaller than that of edge dislocation in both isotropic and anisotropic cases. Therefore, the dislocation in thin film can minimize the total energy either by shortening the dislocation length (i.e., decrease $\alpha$ ) or by aligning its direction approaching to $\vec{b}$ since the screw component has smaller energy factor (i.e., decrease $\beta$ ). The competition between these two mechanisms implies that the final equilibrium dislocation line direction should be located in between the acute angle formed by $\vec{b}$ and $\vec{n}_{1}$.

The equilibrium condition Eq. (3.3) for the projected inclination angle $\alpha$ in both static and dynamic simulations in an anisotropic medium becomes a nonlinear equation

$$
\frac{1+k}{1-k} \sin \alpha-\sin (2 \beta+\alpha)-\sin (2 \beta) \cos \alpha=0
$$

The results of $\alpha$ and $\beta$ obtained from the continuum description in Eq. (3.5) are shown in Fig. 4(a) and (b), respectively. Excellent agreement can be seen between our analytical predictions in anisotropic cases and the results from full molecular simulations as shown in the figure.

Note that in the results of MD simulations shown in the previous section, the obtained instantaneous inclination angle only deviates slightly from its static equilibrium value. Here we can also obtain the same conclusion from theoretical point of view that Eq. (3.5) should be satisfied in both static and dynamic simulations. In fact, once this nonlinear equation does not hold during the dynamic process, according to the force expression in Ref. [42], the force acting on a point approaching to the intersection point will be divergent, and this very large image effect will force the instantaneous inclination angle in dynamic process approach to the value in the static equilibrium case. 


\section{Dynamics of the dislocation}

In this section, in order to further investigate the boundary condition in DD simulations, we consider the dynamic of a dislocation line in thin film and derive a simplified model based upon the Onsager's principle [50-56]. In Onsager's variational principle, a system in which the inertia forces can be neglected is described by some state variables $x=\left\{x_{1}, x_{2}, \cdots, x_{n}\right\}$, and evolution of the system is described by the time derivative of state variables, i.e., $\dot{x}=\left\{\dot{x}_{1}, \dot{x}_{2}, \cdots, \dot{x}_{n}\right\}$. The Rayleighian is then defined by $R(x, \dot{x})=\Phi(\dot{x})+\dot{F}(x)$, where $F(x)$ is the free energy of the system, and $\Phi(\dot{x})=\frac{1}{2} \sum_{i, j} \rho_{i j} \dot{x}_{i} \dot{x}_{j}$ is the dissipation function in which $\rho_{i j}$ are the linear response coefficients. The rates of the state variables are given by minimizing the Rayleighian with respect to $\dot{x}$, i.e., $\delta[\Phi(\dot{x})+\dot{F}(x)]=$ $\sum_{i=1}^{n}\left(\frac{\partial \Phi}{\partial \dot{x}_{i}}+\frac{\partial F}{\partial x_{i}}\right) \delta \dot{x}_{i}=0$.

The first step in developing the simplified model is to choose some appropriate state variables. The results of MD simulations obtained in the previous section show that the dislocation in the dynamics process can be approximated by a straight line except in the vicinity of free surface. Therefore a straight line approximation can be used to model the movement of the dislocation in the system shown in Fig. 1. In fact, the movement of the straight line can be considered as a combination of translation of the center of this straight line and rigid rotation around this center. Thus the movement of the straight dislocation line can be described by two variables: $\alpha$, i.e., the inclination angle of the dislocation lines, and $x_{c}$ which is the displacement of the center of the dislocation line.

The total free energy of the system as shown in Fig. 1 can be divided into two parts: the total stored elastic energy $E_{\text {tot }}$ and the work done by the external stress $W$.

We first give the expression of the total stored elastic energy $E_{\text {tot }}$. Since the dislocation is approximated by a straight line, its elastic energy can be written as [1]

$$
E_{\mathrm{tot}}=E_{0} L \log \left(\frac{L}{e \rho}\right),
$$

where $E_{0}$ is the prelogarithmic energy factor, $L=H / \sin \theta_{\text {inc }}$ is the total length of the dislocation line with $H$ being the width of simulation cell, and $\rho$ is the cut-off parameter associated with dislocation core. The derivative of the total stored elastic energy with respect to the projected inclination angle $\alpha$ is

$$
\frac{d E_{\text {tot }}}{d \alpha}=L \log \left(\frac{L}{e \rho}\right)\left(\frac{d E_{0}}{d \alpha}+\frac{E_{0} \tan \alpha \log \left(\frac{L}{e \rho}\right)}{1+\log \left(\frac{L}{e \rho}\right)}\right) .
$$

Since the length of the dislocation is much larger than the dislocation core cut-off parameter $\rho$ (i.e., $L \gg \rho$ ), we have $\log (L / e \rho) \gg 1$, and the derivative of the total elastic energy with respect to the projected inclination angle $\alpha$ could be approximated by:

$$
\frac{d E_{\mathrm{tot}}}{d \alpha} \approx L \log \left(\frac{L}{e \rho}\right)\left(\frac{d E_{0}}{d \alpha}+E_{0} \tan \alpha\right) .
$$


The variation of the rate of the elastic contribution in the total free energy can be obtained based upon the Onsager's principle:

$$
\delta \dot{E}_{\text {tot }}=L \log \left(\frac{L}{e \rho}\right)\left(\frac{d E_{0}}{d \alpha}+E_{0} \tan \alpha\right) \delta \dot{\alpha} .
$$

The variation of the rate of the work done by the external applied stress $\sigma^{\text {ext }}$ on the dislocation in thin film system can be written as

$$
\delta \dot{W}=-\int_{\Gamma}\left[\left(\sigma^{\mathrm{ext}} \cdot \overrightarrow{\boldsymbol{b}}\right) \times \overrightarrow{\boldsymbol{\xi}}\right] \cdot \delta \overrightarrow{\boldsymbol{v}} d l,
$$

where $\delta \overrightarrow{\boldsymbol{v}}=\delta \dot{x}_{c} \hat{\boldsymbol{x}}_{c}+l \delta \dot{\alpha} \hat{\boldsymbol{\alpha}}$ is the velocity of infinitesimal dislocation segment $d l$, in which $\hat{x}_{c}$ is the translation direction of the center of dislocation line and $\hat{\alpha}$ is the unit direction perpendicular to the dislocation line in the slip plane. Therefore $\overrightarrow{\boldsymbol{\xi}} \times \hat{\boldsymbol{x}}_{c}=\cos \alpha \overrightarrow{\boldsymbol{n}}_{s}$ and $\overrightarrow{\boldsymbol{\xi}} \times \hat{\boldsymbol{\alpha}}=$ 0 with $\vec{n}_{s}$ being the normal direction of the slip plane. Thus we have

$$
\delta \dot{W}=-\int_{-L / 2}^{L / 2}\left(\sigma^{\mathrm{ext}} \cdot \overrightarrow{\boldsymbol{b}}\right) \cdot \overrightarrow{\boldsymbol{n}}_{s} \cos \alpha \delta \dot{x}_{c} d l=-f_{\text {slip }} L \cos \alpha \delta \dot{x}_{c},
$$

where $f_{\text {slip }}=\left(\boldsymbol{\sigma}^{\text {ext }} \cdot \overrightarrow{\boldsymbol{b}}\right) \cdot \overrightarrow{\boldsymbol{n}}_{s}$ is the projection of the Peach-Koehler force due to the external stress on the slip plane. The variation of the rate of the total free energy with respect to the rates of two state variables can be obtained from Eq. (4.4) and Eq. (4.6):

$$
\begin{aligned}
& \frac{\delta \dot{F}}{\delta \dot{\alpha}}=L \log \left(\frac{L}{e \rho}\right)\left(\frac{d E_{0}}{d \alpha}+E_{0} \tan \alpha\right), \\
& \frac{\delta \dot{F}}{\delta \dot{x}_{c}}=-f_{\text {slip }} L \cos \alpha .
\end{aligned}
$$

The dissipation function $\Phi\left(\dot{\alpha}, \dot{x}_{c}\right)$ of the dislocation is

$$
\Phi=\int_{-L / 2}^{L / 2} \frac{1}{2} \frac{v^{2}}{M_{d}} d l=\int_{-L / 2}^{L / 2} \frac{\left(\dot{x}_{c}+l \dot{\alpha}\right)^{2}}{2 M_{d}} d l=\frac{L \dot{x}_{c}^{2}}{2 M_{d}}+\frac{L^{3} \dot{\alpha}^{2}}{24 M_{d}},
$$

where $L=H / \sin \theta_{\text {inc }}$ is the length of dislocation line. $v$ is the velocity of dislocation segment, which could be given by $v=M_{d} \cdot f$ in overdamped manner due to the friction, $M_{d}$ is the corresponding dislocation mobility. Therefore the dissipation of infinitesimal dislocation segment is $v^{2} / 2 M_{d}$. This dissipative function leads to $\partial \Phi / \partial \dot{\alpha}=L^{3} \dot{\alpha} / 12 M_{d}$ and $\partial \Phi / \partial \dot{x}_{c}=L \dot{x}_{c} / M_{d}$, and the following evolution equations for $\alpha$ and $x_{c}$ can be derived based upon the Onsager variational principle (i.e., $\delta \Phi+\delta \dot{F}=0$ ) that

$$
\begin{aligned}
\frac{\dot{\alpha}}{M_{d}} & =\frac{12 \sin ^{2} \theta_{\text {inc }}}{H^{2}}\left(\frac{d E_{0}}{d \alpha}+E_{0} \tan \alpha\right) \log \left(\frac{e \rho \sin \theta_{\text {inc }}}{H}\right), \\
\frac{\dot{x}_{c}}{M_{d}} & =f_{\text {slip }} \cos \alpha,
\end{aligned}
$$


where $f_{\text {slip }}=\left(\sigma^{\text {ext }} \cdot \overrightarrow{\boldsymbol{b}}\right) \cdot \overrightarrow{\boldsymbol{n}}_{s}$ is the projection of the Peach-Koehler force due to the external stress on the slip plane, and $H$ is the width of simulation cell. Since the prefactor for the evolution of the inclination angle is usually much greater than the prefactor for the evolution of the center, the initially in-equilibrium dislocation should undergo a very quick rotation stage governed by the first equation, and then when the inclination angle approaches to the value in the corresponding static case, the evolution of dislocation is governed by the second equation. The above equations also show that the evolution of center position and the inclination angle can be decoupled.

We compare the results obtained by using this simplified model and the MD simulations for simulation samples with surface normal $\vec{n}=[201]$ under different values of the applied strain $\varepsilon_{0}=0.25 \%$ and $\varepsilon_{0}=0.5 \%$. We use the same parameters in both cases (where the mobility coefficient $M_{d}$ and the core radius $\rho$ are obtained by fitting from the MD simulations for $\varepsilon_{0}=0.25 \%$ ). Comparisons of results using these two methods are shown in Fig. 6(a) and (b). It can be seen that the predicted positions of the dislocation using the simplified model agree perfectly with the positions of the dislocation obtained in MD simulations indicated by the region formed by dislocation core atoms. We further calculate the positions of the upper intersection point, the center point and the lower intersection point using our simplified model and compare them with the positions of corresponding points in MD simulations, and the comparisons are shown in Fig. 6(c1) and (c2). The excellent agreement between our simplified model's predictions and the MD simulation results shown in Fig. 6(a)-(c) from the very beginning of the simulations validates our simplified model.

Here we have demonstrated that this simplified model is valid in the simple case that there are only one single threading dislocation and no other defects and intersections. However, this simplified model can be used to describe more complicated dynamic cases in the manner as the boundary condition of a dislocation in the vicinity of free surface and is coupled with DD simulations in the bulk. More precisely, each dislocation can be decomposed into two parts: one part is the portion away from the free surface and its motion is described by standard DD simulation; another part is the dislocation segment in the vicinity of free surface, and its motion is approximated by the simplified model with two parameters, i.e., the inclination angle and the center of the straight segment (or one ending point of the straight segment). Generally speaking, this simplified model can be used as the boundary condition in the vicinity of the intersection point between a dislocation and the free surface in DD simulations with presence of free surfaces.

\section{Conclusions and discussion}

In summary, we have systematically studied the inclination angle $\theta_{\text {inc }}$ between the dislocation and free surface by using both molecular static and dynamic simulations in BCC metal $\alpha$-iron. We have found that the equilibrium dislocations are straight in simulation cells with initial edge/mixed dislocations. However, in the some simulation cells with 
initial screw dislocations, the final dislocation will break into two straight segments. The inclination angle obtained in dynamic simulations quickly converges to the corresponding value in static simulations.

Furthermore, we derived an analytical expression for projected inclination angle as shown in Eq. (3.5) in which the inclination angle can be uniquely determined by the angle between the projected normal and the Burgers vector. The analytical expression is validated by excellent agreement in results compared with the molecular static and dynamic simulations. This more general expression can be applied in anisotropic medium as well as when the slip plane is inclined with the surface, thus it can be used as a boundary condition in dislocation dynamic simulations to describe the interception angle of a dislocation line with respect to the free surface. We have further developed an accurate simplified model to describe the movement of dislocation in thin film based upon Onsager's variational principle and straight line approximation observed from MD simulations, which can be used as the boundary condition in the vicinity of the intersection point between a dislocation and the free surface in DD simulations with presence of free surfaces.

\section{Acknowledgments}

This work is supported by National Natural Science Foundation of China (Grant Nos. 12071362, 12071363, 11671312, 11701433), the National Key Research and Development Program of China (No. 2020YFA0714200) and the Natural Science Foundation of Hubei Province (No. 2019CFA007). The work of YX was supported by the Hong Kong Research Grants Council General Research Fund (No. 16307319). The numerical calculations in this paper have been done on the supercomputing system in the Supercomputing Center of Wuhan University.

\section{References}

[1] Hirth, J.P., Lothe, J., 1982. Theory of Dislocations, 2nd ed. John Wiley, New York.

[2] Bulatov, V.V., Cai, W., 2006. Computer Simulations of Dislocations. Oxford.

[3] Sills, R., Kuykendall, W.P., Aghaei, A., Cai, W., 2016. Fundamentals of dislocation dynamics simulations, in: C, C.R.W., Tucker, G.J. (Eds.), Multiscale Materials Modeling for Nanomechanics. Springer Series in Materials Science, vol 245.. Springer, Cham. chapter 2, pp. 53-87.

[4] Dewit, R., 1968. Thermodynamic force on a dislocation. Journal of Applied Physics 39, 137.

[5] Zhou, Q., Wang, J., Misra, A., Huang, P., Wang, F., Xu, K., 2017. Dislocations interaction induced structural instability in intermetallic $\mathrm{Al}_{2} \mathrm{Cu}$. npj Computational Materials 3, 24.

[6] Kraych, A., Clouet, E., Dezerald, L., Ventelon, L., Willaime, F., Rodney, D., 2019. Non-glide effects and dislocation core fields in BCC metals. npj Computational Materials 5, 109.

[7] Moriarty, J.A., Vitek, V., Bulatov, V.V., Yip, S., 2002. Atomistic simulations of dislocations and defects. Journal of Computer-aided Materials Design 9, 99.

[8] Lepinoux, J., Kubin, L.P., 1987. The dynamic organization of dislocation structures: A simulation. Scr. Metall. Mater 21, 833. 
[9] Ghoniem, N.M., Amodeo, R., 1988. Computer simulaltion of dislocation pattern formation. Solid State Phenom. 3\&4, 377.

[10] Gulluoglu, A.N., Srolovitz, D.J., LeSar, R., Lomdahl, P.S., 1989. Dislocation distributions in two dimensions. Scr. Metall. 23, 1347.

[11] Kubin, L.P., Canova, G., Condat, M., DeVincre, B., Pontikis, V., Brechet, Y., 1992. Dislocation microstructures and plastic flow: A 3D simulation. Solid State Phenom. 23, 455.

[12] DeVincre, B., Kubin, L.P., 1994. Simulations of forest interactions and strain hardening in FCC crystals. Modell. Simul. Mater. Sci. Eng. 2, 559.

[13] der Giessen, E.V., Needleman, A., 1995. Discrete dislocation plasticity: A simple planar model. Modell. Simul. Mater. Sci. Eng. 3, 689-735.

[14] Schwarz, K.W., 1999. Simulation of dislocations on the mesoscopic scale. I. methods and examples. J. Appl. Phys. 85, 108.

[15] Fivel, M.C., Canova, G.R., 1999. Developing rigorous boundary conditions to simulations of discrete dislocation dynamics. Modell. Simul. Mater. Sci. Eng. 7, 753.

[16] Ghoniem, N.M., Tong, S.H., Sun, L.Z., 2000. Parametric dislocation dynamics: A thermodynamics-based approach to investigations of mesoscopic plastic deformation. Phys. Rev. B 61, 913.

[17] Lemarchand, C., Devincre, B., Kubin, L.P., 2001. Homogenization method for a discretecontinuum simulation of dislocation dynamics. J. Mech. Phys. Solids 49, 1969-1982.

[18] Weygand, D., Friedman, L.H., der Giessen, E.V., Needleman, A., 2002. Aspects of boundaryvalue problem solutions with three-dimensional dislocation dynamics. Modell. Simul. Mater. Sci. Eng. 10, 437.

[19] Zbib, H.M., de la Rubia, T.D., 2002. A multiscale model of plasticity. Int. J. Plast. 18, 11331163.

[20] Xiang, Y., Cheng, L.T., Srolovitz, D.J., E, W., 2003. A level set method for dislocation dynamics. Acta Mater. 51, 5499.

[21] Liu, X.H., Scwarz, K.W., 2005. Modelling of dislocations intersecting a free surface. Modelling and Simulation in Materials Science and Engineering 13, 1233.

[22] Bulatov, V.V., Hsiung, L.L., Tang, M., Arsenlis, A., Bartelt, M.C., Cai, W., Florando, J.N., Hiratani, M., Rhee, M., Hommes, G., Pierce, T.G., de la Rubia, T.D., 2006. Dislocation multijunctions and strain hardening. Nature 440, 1174-1178.

[23] Devincre, B., Kubin, L., Hoc, T., 2006. Physical analyses of crystal plasticity by DD simulations. Scr. Mater. 54, 741-746.

[24] Espinosa, H.D., Panico, M., Berbenni, S., Schwarz, K.W., 2006. Discrete dislocation dynamics simulations to interpret plasticity size and surface effects in freestanding FCC thin films. Int. J. Plast. 22, 2091-2117.

[25] Tang, M., Cai, W., Xu, G., Bulatov, V.V., 2006. A hybrid method for computing forces on curved dislocations intersecting free surfaces in three-dimensional dislocation dynamics. Modelling and Simulation in Materials Science and Engineering 14, 1139.

[26] Wang, Z., Ghoniem, N.M., Swaninarayan, S., LeSar, R., 2006. A parallel algorithm for 3D dislocation dynamics. J. Comput. Phys. 219, 608.

[27] Quek, S., Xiang, Y., Zhang, Y., Srolovitz, D., Lu, C., 2006. Level set simulation of dislocation dynamics in thin films. Acta Mater. 54, 2371-2381.

[28] Arsenlis, A., Cai, W., Tang, M., Rhee, M., Oppelstrup, T., Hommes, T.G., Pierce, T.G., Bulatov, V.V., 2007. Enabling strain hardening simulations with dislocation dynamics. Modell. Simul. Mater. Sci. Eng. 15, 553.

[29] El-Awady, J.A., Biner, S.B., Ghoniem, N.M., 2008. A self-consistent boundary element, para- 
metric dislocation dynamics formulation of plastic flow in finite volumes. J. Mech. Phys. Solids 56, 2019-2035.

[30] Queyreau, S., Monnet, G., Devincre, B., 2009. Slip systems interactions in alpha-iron determined by dislocation dynamics simulations. Int. J. Plast. 25, 361-377.

[31] Weinberger, C.R., Aubry, S., Lee, S.W., Nix, W.D., Cai, W., 2009. Modelling dislocations in a free-standing thin film. Modell. Simul. Mater. Sci. Eng. 17, 075007.

[32] Devincre, B., Madec, R., Monnet, G., Queyreau, S., Gatti, R., Kubin, L., 2011. Modeling crystal plasticity with dislocation dynamics simulations: The 'micro-megas' code. Mech. Nano-objects, 81-100.

[33] Zhou, C., Beyerlein, I.J., LeSar, R., 2011. Plastic deformation mechanisms of FCC single crystals at small scales. Acta Mater. 59, 7673-7682.

[34] Zhao, D., Huang, J., Xiang, Y., 2012. Fast multipole accelerated boundary integral equation method for evaluating the stress field associated with dislocations in a finite medium. Commun. Comput. Phys. 12, 226-246.

[35] Huang, M., Zhao, L., Tong, J., 2012. Discrete dislocation dynamics modelling of mechanical deformation of nickel-based single crystal superalloys. Int. J. Plasticity 28, 141.

[36] Po, G., Mohamed, M.S., Crosby, T., Erel, C., El-Azab, A., Ghoniem, N., 2012. Recent progress in discrete dislocation dynamics and its applications to micro plasticity. JOM: The journal of the Minerals, Metals and Materials Society 66, 2108-2120.

[37] Zhou, C., LeSar, R., 2012. Dislocation dynamics simulations of plasticity in polycrystalline thin films. Int. J. Plast. 30, 185-201.

[38] Devincre, B., 2013. Dislocation dynamics simulations of slip systems interactions and forest strengthening in ice single crystal. Philos. Mag. 93, 235-246.

[39] Ferroni, F., Tarleton, E., Fitzgerald, S., 2014. Dislocation dynamics modelling of radiation damage in thin films. Modell. Simul. Mater. Sci. Eng. 22, 045009.

[40] Vattré, A., Devincre, B., Feyel, F., Gatti, R., Groh, S., Jamond, O., Roos, A., 2014. Modelling crystal plasticity by 3D dislocation dynamics and the finite element method: The discretecontinuous model revisited. J. Mech. Phys. Solids 63, 491-505.

[41] Bertin, N., 2019. Connecting discrete and continuum dislocation mechanics: A non-singular spectral framework. Int. J. Plast. 122, 268-284.

[42] Lothe, J., 1970. The image force on dislocations at free surfaces - Comments on the concept of line tension. Fundamental Aspects of Dislocation Theory Spec. Publ. 317, 11.

[43] Yoffe, E.H., 1961. A dislocation at a free surface. Philos. Mag. 6, 1147-1155.

[44] Mendelev, M.I., Han, S., Srolovitz, D.J., Ackland, G.J., Sun, D.Y., Asta, M., 2003. Development of new interatomic potentials appropriate for crystalline and liquid iron. Philosophical Magazine 83, 3977.

[45] Domain, C., Monnet, G., 2005. Simulation of screw dislocation motion in iron by molecular dynamics simulations. Physical Review Letters 95, 215506.

[46] Clouet, E., Garruchet, S., Nguyen, H., Perez, M., Becquart, C.S., 2008. Dislocation interaction with $\mathrm{C}$ in $\alpha$-Fe: A comparison between atomic simulations and elasticity theory. Acta Materialia 56, 3450.

[47] Queyreau, S., Marian, J., Gilbert, M.R., Wirth, B.D., 2011. Edge dislocation mobilities in bcc Fe obtained by molecular dynamics. Physical Review B 84, 064106.

[48] Peng, S., Wei, Y., Jin, Z., Yang, W., 2019. Supersonic screw dislocations gliding at the shear wave speed. Physical Review Letters 122, 045501.

[49] Eshelby, J.D., 1949. LXXXII. Edge dislocations in anisotropic materials. The London, Edinburgh, and Dublin Philosophical Magazine and Journal of Science 40:308, 903. 
[50] Onsager, L., 1931a. Reciprocal relations in irreversible processes. I. Physical Review 37(4), 405.

[51] Onsager, L., 1931b. Reciprocal relations in irreversible processes. II. Physical Review 38(12), 2265.

[52] Qian, T., Wang, X.P., Sheng, P., 2006. A variational approach to moving contact line hydrodynamics. J. Fluid Mech. 564, 333-360.

[53] Xu, X., Qian, T., 2017. Hydrodynamics boundary conditions derived from Onsager's variational principle. Procedis IUTAM 20, 144-151.

[54] Xu, X., Di, Y., Doi, M., 2016. Variational method for liquids moving on a substrate. Phys. Fluids 28(8), 087101.

[55] Di, Y., Xu, X., Zhou, J., Doi, M., 2018. Thin film dynamics in coating problems using Onsager principle. Chin. Phys. 27(2), 024501.

[56] Man, X., Doi, M., 2016. Ring to mountain transition in deposition pattern of drying droplets. Phys. Rev. Lett. 116(6), 066101.

[57] OKraft, O., Gruber, P. A., Mnig, R., Weygand, D., 2010. Plasticity in confined dimensions. Annual Review of Materials Research 40, 293. 